\title{
New constraints on interacting dark energy from cosmic chronometers
}

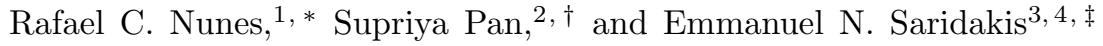 \\ ${ }^{1}$ Departamento de Física, Universidade Federal de Juiz de Fora, 36036-330, Juiz de Fora, MG, Brazil \\ ${ }^{2}$ Department of Physical Sciences, Indian Institute of Science Education \\ and Research - Kolkata, Mohanpur - 741246, West Bengal, India \\ ${ }^{3}$ Instituto de Física, Pontificia Universidad de Católica de Valparaíso, Casilla 4950, Valparaíso, Chile \\ ${ }^{4}$ CASPER, Physics Department, Baylor University, Waco, TX 76798-7310, USA
}

\begin{abstract}
We use the latest compilation of observational Hubble parameter measurements estimated with the differential evolution of cosmic chronometers, in combination with the local value of the Hubble constant recently measured with $2.4 \%$ precision, to constrain the cosmological scenario where dark energy interacts directly with the dark matter sector. To diminish the degeneracy between the parameters we additionally consider standard probes, such as Supernovae Type Ia from joint light curves (JLA) sample, Baryon Acoustic Oscillation distance measurements (BAO), and cosmic microwave background data from Planck 2015 estimations. Our analysis shows that the direct interaction between dark energy and dark matter is mildly flavored, while the dark energy equationof-state parameter is $w<-1$ at $3 \sigma$ confidence level.
\end{abstract}

PACS numbers: 98.80.-k, 95.36.+x, 95.35.+d, 98.80.Es

\section{INTRODUCTION}

According to latest observations 1, around $69 \%$ of the universe is constituted by the dark energy (DE) sector, responsible for its current accelerating phase, and almost $26 \%$ by the cold dark matter $(\mathrm{CDM})$ sector in the form of dust. These two sectors are considered not to interact with each other, and the resulting scenario has been believed to be the best description of the present observable universe, due to its agreement with a large number of independent observations. However, some potential problems such as the coincidence one [2, namely why are the dark energy and dark matter energy densities of the same order although they follow completely different evolutions, have led to a large amount of research towards scenarios in which the above two dark sectors exhibit a direct interaction. Such a mutual direct interaction between dark energy and dark matter cannot be excluded from the field theoretical point of view, independently of the specific nature of the former, i.e. whether it arises through a universe-content modification 3, 4, or through a gravitational modification [5, 6. Hence, many different scenarios of interacting dark energy have been constructed (see [7, 8, for reviews and references therein). Interestingly enough, recently it has been shown that the current data might favor the late-time interaction between CDM and the vacuum energy [9-12] or the dynamical dark energy sector 13,23 .

In the present work we are interested in providing updated constraints on the scenarios of interacting dark energy, using the very recent observational data, along with the new local value of the Hubble parameter with a $2.4 \%$

\footnotetext{
*Electronic address: nunes@ecm.ub.edu

${ }^{\dagger}$ Electronic address: span@iiserkol.ac.in

‡Electronic address: Emmanuel_Saridakis@baylor.edu
}

determination, released in [41. In particular, considering a direct interaction between dark energy and dark matter in a Friedmann-Robertson-Walker (FRW) background, and assuming that both (effective) fluids obey a barotropic equation of state, the cosmological dynamics becomes richer and thus the evolution equations for CDM and dark energy become different from their standard forms. Hence, one can use observational data in order to fit the deviation from non-interaction, without the need to specify a specific interaction form, i.e. keeping the analysis in a general ground. The manuscript is organized as follows: In Section [I] we provide a brief description of the expansion history of the universe in the background of an interacting scenario. In Section III we describe the latest data sets for our analysis, while in Section IV we use them and we extract the constraints on the various quantities. Finally, Section $\mathrm{V}$ is devoted to summary and conclusions.

\section{INTERACTING DARK ENERGY}

Let us briefly describe the scenario of interacting dark energy. In a background of a FRW universe with metric $d s^{2}=-d t^{2}+a(t)^{2}\left[d r^{2} /\left(1-k r^{2}\right)+r^{2}\left(d \theta^{2}+\sin ^{2} \theta d \phi^{2}\right)\right]$, where $a(t)$ is the scale factor and $k$ the spatial curvature (with $k=0,-1,+1$ for flat, open and closed universe respectively), the first Friedmann equation can be written as

$$
H^{2}+\frac{k}{a^{2}}=\frac{8 \pi G}{3}\left(\rho_{\gamma}+\rho_{b}+\rho_{c d m}+\rho_{d e}\right),
$$

where $H=\dot{a} / a$ is the Hubble rate, with dots denoting derivatives with respect to the cosmic time, and with $\rho_{\gamma}, \rho_{b}, \rho_{c d m}$, and $\rho_{d e}$ denoting the energy densities of radiation, baryons, cold dark matter and dark energy, respectively. 
Since the physics of the radiation and baryonic sector is known quite well, we assume that these sectors are independently conserved. On the other hand, if the dark energy and dark matter sectors interact directly, the energy conservation law for the interacting CDM-DE components reads as $u_{\alpha} T_{; \beta}^{\alpha \beta}=0$, where the total energymomentum tensor is $T^{\alpha \beta}=T_{c d m}^{\alpha \beta}+T_{d e}^{\alpha \beta}$, or equivalently [7, 8.

$$
\dot{\rho}_{c d m}+3 \frac{\dot{a}}{a} \rho_{c d m}=-\dot{\rho}_{d e}-3 \frac{\dot{a}}{a}\left(\rho_{d e}+p_{d e}\right)=Q,
$$

where $Q$ is a specific interaction form that has to be chosen by hand. As usual, one can quantify the effect of the above interaction on the dark matter evolution through a deviation from the standard dust-matter evolution, namely considering that the dark matter energy density evolves as $24,27$.

$$
\rho_{c d m}=\rho_{c d m, 0} a^{-3+\delta},
$$

with $\rho_{c d m, 0}$ the present value, and where the parameter $\delta$ becomes zero in the non-interacting case. Hence, the parameter $\delta$ quantifies the deviation from non-interacting case $(\delta<0$ corresponds to energy flow from dark matter to dark energy), without the need to consider a specific interaction form $Q$. Combining (2), (3) and considering that the dark energy sector is described by an equationof-state parameter of the form $w=p_{d e} / \rho_{d e}$, we find that the energy density of the dark energy component evolves as

$$
\rho_{d e}=\rho_{d e, 0} a^{-3(1+w)}+\frac{\delta \rho_{c d m, 0}}{3|w|-\delta}\left[a^{-3+\delta}-a^{-3(1+w)}\right],
$$

where $\rho_{d e, 0}$ is the present value of $\rho_{d e}$. Clearly, in the absence of a coupling with the CDM component, the conventional dynamical dark energy scenario is recovered. Note that for $w=-1$ and $\delta \neq 0$, we may identify $\rho_{d e}=\rho_{\text {vacuum }}$ and the above expressions reduce to the vacuum decaying scenario [28 34].

\section{CURRENT OBSERVATIONAL CONSTRAINTS}

In the present work we confront the constraints on the cosmological parameters that can be obtained by probes that map the late-time universe $(z<2.36)$ expansion history. The baseline of our analysis is the Hubble parameter measurements obtained with the cosmic chronometers (CC) technique, however we additionally consider standard probes such as Supernovae Type Ia (SNe Ia), local Hubble parameter value $H_{0}$ ones, and Baryon Acoustic Oscillation distance measurements $(\mathrm{BAO})$, to diminish the degeneracy between the free parameters of the models. In the following subsections, we present the data sets considered in our analysis.

\section{A. Cosmic chronometer dataset and local value of the Hubble constant}

The CC approach to measure $H(z)$ was first introduced in [35, and it uses relative ages of the most massive and passively evolving galaxies to measure $d z / d t$, from which $H(z)$ is inferred. The latest implementation has been explained in detail in [36, where the possible sources of uncertainty and related issues are also discussed. We consider the compilation of Hubble parameter measurements provided by [36, 37. It contains the latest updated list of $H(z)$ measurements [36 40 ] obtained with the cosmic chronometers approach, comprising of 30 measurements spanning the redshift range $0<z<2$. This sample covers roughly 10 Gyr of cosmic time. Furthermore, in our analysis we also include the new local value of $H_{0}$ as measured by [41] with a $2.4 \%$ determination, which yields $H_{0}=73.02 \pm 1.79 \mathrm{~km} / \mathrm{s} / \mathrm{Mpc}$.

\section{B. Type Ia Supernovae}

SNe Ia were the main tool to discover late-time acceleration [42, 43, and they still provide the best constraints on dark energy sector. SNe Ia are very bright "standard candles" and thus are used to measure cosmic distances. We consider here the latest "joint light curves" (JLA) sample 44, comprised of 740 SNe Ia in the redshift range $z \in[0.01,1.30]$. From the observational point of view, the distance modulus of a SNe Ia can be abstracted from its light curve assuming that supernovae with identical color, shape and galactic environment have on average the same intrinsic luminosity for all redshifts. This hypothesis is quantified by a empirical linear relation, yielding a standardized distance modulus $\mu=5 \log _{10}\left(d_{L} / 10 p c\right)$ of the form

$$
\mu=m_{B}^{*}-\left(M_{B}-\alpha \times X_{1}+\beta \times C\right),
$$

where $m_{B}^{*}$ corresponds to the observed peak magnitude in rest frame $\mathrm{B}$ band and $\alpha, \beta$, and $M_{B}$ are nuisance parameters in the distance estimate. The absolute magnitude is related to the host stellar mass $\left(M_{\text {stellar }}\right)$ by a simple step function: $M_{B}=M_{B}$ if $M_{\text {stellar }}<10^{10} M_{\odot}$, otherwise $M_{B}=M_{B}+\Delta_{M}$. The light-curve parameters $\left(m_{B}^{*}, X_{1}\right.$, and $\left.C\right)$ result from the fit of a model of the SNe Ia spectral sequence to the photometric data. In our analysis we assume $M_{B}, \Delta_{M}, \alpha$ and $\beta$ as nuisance parameters.

\section{Baryon Acoustic oscillation}

Another important cosmological probe are the baryon acoustic oscillations (BAO). The $\mathrm{BAO}$ can be traced to pressure waves at the recombination epoch, generated by cosmological perturbations in the primeval baryonphoton plasma, appearing as distinct peaks in large angular scales. We use the following $\mathrm{BAO}$ data to constrain 
the expansion history of the scenario at hand: the measurement from the Six Degree Field Galaxy Survey (6dF) [45, the Main Galaxy Sample of Data Release 7 of Sloan Digital Sky Survey (SDSS-MGS) [46], the LOWZ and CMASS galaxy samples of the Baryon Oscillation Spectroscopic Survey (BOSS-LOWZ and BOSS-CMASS, respectively) [47, and the distribution of the LymanForest in BOSS (BOSS-Ly) [48. These measurements and their corresponding effective redshift $z$ are summarized in Table 【.

\begin{tabular}{|c|c|c|c|c|}
\hline Survey & $z$ & Parameter & Measurement & Reference \\
\hline $6 \mathrm{dF}$ & 0.106 & $r_{s} / D_{V}$ & $0.327 \pm 0.015$ & 45 \\
\hline SDSS-MGS & 0.10 & $D_{V} / r_{s}$ & $4.47 \pm 0.16$ & [46] \\
\hline BOSS-LOWZ & 0.32 & $D_{V} / r_{s}$ & $8.47 \pm 0.17$ & 47 \\
\hline BOSS-CMASS & 0.57 & $D_{V} / r_{s}$ & $13.77 \pm 0.13$ & 47] \\
\hline BOSS- $L y_{\alpha}$ & 2.36 & $c /\left(H r_{s}\right)$ & $9.0 \pm 0.3$ & 48 \\
\hline BOSS- $L y_{\alpha}$ & 2.36 & $D_{A} / r_{s}$ & $10.08 \pm 0.4$ & 48 \\
\hline
\end{tabular}

TABLE I: Baryon acoustic oscillation (BAO) data measurements included in our analysis.

\section{Cosmic microwave background}

Cosmic microwave background (CMB) radiation provides a unique window to understand the structure formation in a given cosmological model. In order to avoid the calculation of the evolution of the linear density perturbations, and incorporate as much empirical information as possible, one can develop a substitution for the full Boltzmann analysis of CMB. The fundamental principle uses certain characteristic distance scales to summarize the CMB data, namely the shift parameter $R$ [49, 50] that determines the amplitude of acoustic peaks in the power spectrum of CMB temperature anisotropy, and the acoustic scale $l_{a}$ that determines the acoustic peak structure [51. In this work we use the distance measurements $R$ and $l_{a}$, obtained in 52 by using the Markov Chain Monte Carlo chains from Planck TT, TE, EE + lowP data in Planck Legacy Archive. We use the measurements marginalized over the amplitude of the lensing power spectrum.

Let us now describe how a coupled dark energy affects the anisotropies of the cosmic microwave background radiation and the matter spectrum. We follow [55, 56, where a synchronous gauge has been adopted, and thus the line element of the linearly perturbed FRW metric can be written as

$$
d s^{2}=-d t^{2}+a^{2}(t)\left(\delta_{i j}+h_{i j}\right) d x^{i} d x^{j},
$$

where $h_{i j}$ denotes the metric perturbation. For simplicity we restrict to the scalar modes $h$ and $\eta$ of the metric perturbations, where $h, \eta$ are respectively the trace and traceless parts of the metric $h_{i j}$, which in Fourier space read as

$$
\begin{aligned}
h_{i j}(x, \tau)=\int d^{3} k \exp ^{i \vec{k} \cdot \vec{x}}\left[\hat{k}_{i} \hat{k}_{j} h(\vec{k}, \tau)\right. \\
\left.+\left(k_{i} k_{j}-\frac{1}{3} \delta_{i j}\right) 6 \eta(\vec{k}, \tau)\right],
\end{aligned}
$$

with $k$ the wavenumber of the Fourier mode $(\vec{k}=k \hat{k})$. We assume that the dark energy perturbation in the dark matter comoving frame is identically zero. Hence, in an interacting dark energy - dark matter system, the perturbed part of the energy momentum for the dark matter evolution writes as [55, 56]:

$$
\dot{\delta}_{c d m}-\frac{Q}{\rho_{m}} \delta_{c d m}-\frac{k^{2}}{a^{2}} \theta_{c d m}+\frac{\dot{h}}{2}=0,
$$

where $\delta_{c d m}$ is the total density perturbation of cold dark matter, with $\theta_{c d m}$ its total covariant velocity perturbation, while the interaction term $Q$ can be obtained through (2) and (3).

As usual we work in a comoving synchronous gauge, in which the dark matter velocity is zero. The baryon component is conserved independently, and the perturbation equations for the baryon density contrast and velocity respectively write as $\dot{\delta_{b}}-\frac{k^{2}}{a^{2}} \theta_{b}=-\frac{\dot{h}}{2}$ and $\dot{\theta}_{b}=0$. Finally, photons and neutrinos are also conserved and follow the standard evolution equation [57.

\section{RESULTS}

We now proceed to the main analysis, namely to use the above data sets in order to constrain the scenario of interacting dark energy described in Section III In order to fit the free parameters of the scenario we use the public code CLASS [53] in interface with the public Monte Carlo code Monte Python [54. Moreover, we choose the Metropolis Hastings algorithm as our sampling method.

In Figures 1 and 2 we respectively depict the theoretical predictions for the CMB temperature power spectrum and for the linear matter power spectrum, for the present interacting dark energy-dark matter model, as well as for the flat $\Lambda \mathrm{CDM}$ scenario. From the CMB temperature power spectrum we deduce that the coupling between dark matter - dark energy affects the microwave background temperature anisotropies at large angular scales, especially at very low $l$ (in particular for $l<30$ ). Furthermore, significant effects on the linear matter power spectrum are also observed at large scales (for $k<0.034$ $\mathrm{h} / \mathrm{Mpc})$.

Additionally, in Tables II and III we summarize the main results of our statistical analysis, and in Figures 3 and 4 we present the contour plots for the free parameters of the scenario of interacting dark energy, using $C C$ $+H_{0}$ and $C C+H_{0}+\mathrm{SNeIa} / \mathrm{JLA}+\mathrm{BAO}+\mathrm{CMB}$ observations, respectively. 


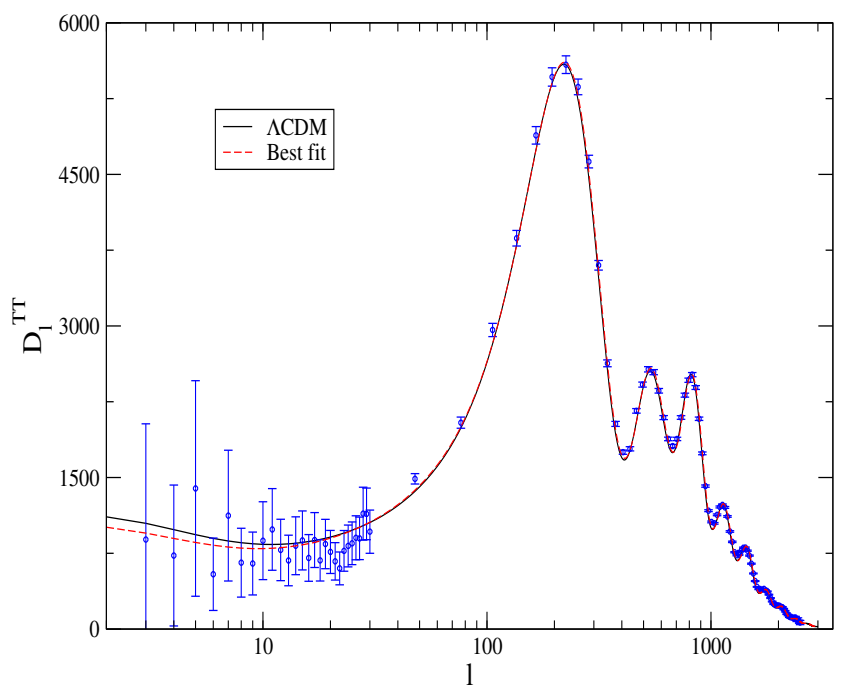

FIG. 1: The CMB TT power spectrum $D_{l}^{T T}=l(l+$ 1) $C_{l} / 2 \pi \mu K^{2}$, for the interacting scenario at hand (dashedred line) and for the flat $\Lambda C D M$ cosmology (black-solid line). The data with their error bars have been taken from Planck Collaboration [58].

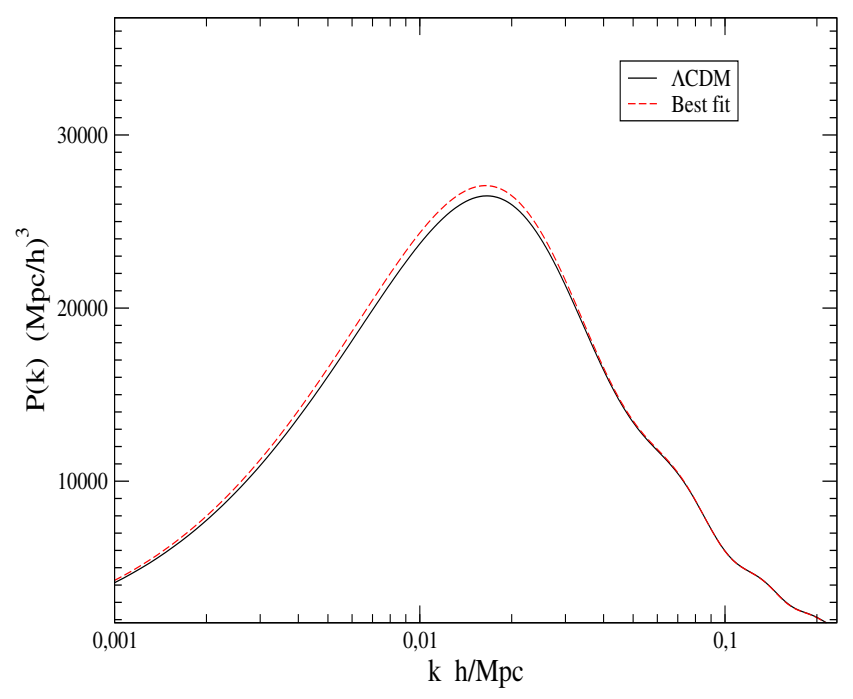

FIG. 2: The theoretical prediction for linear matter power spectrum, for the interacting scenario at hand (dashed-red line) and for the flat $\Lambda$ CDM cosmology (black-solid line).

As we can see, when analyzing the scenario with $C C+$ $H_{0}$ data, we observe that $\delta \neq 0$ combined with $w<-1$ is preferred (see Table II). On the other hand, when we break the degeneracy of the parameters, combining the analysis with SNeIa/JLA + BAO + CMB observations, namely using $C C+H_{0}+\mathrm{SNe} \mathrm{Ia}+\mathrm{BAO}+\mathrm{CMB}$ data, we observe that $\delta \simeq 0$ (with a minor tendency for $\delta \gtrsim 0$, i.e. for an energy flow from dark energy to dark matter), however with $w<-1$ up to $3 \sigma$ confidence level. More specifically we note that $-1.169 \leq w \leq-1.009$ at $3 \sigma$. The $2 \sigma$ confidence-level bounds can be seen in Table III.

Hence, we deduce that the global fits from the map of

\begin{tabular}{ccccc}
\hline \hline Param. best-fit & mean $\pm \sigma$ & $95 \%$ lower & $95 \%$ upper \\
\hline \hline$\Omega_{c d m 0}$ & 0.386 & $0.3839_{-0.094}^{+0.098}$ & 0.2045 & 0.5634 \\
$H_{0}$ & 72.67 & $72.95_{-1.8}^{+2.1}$ & 68.93 & 76.71 \\
$\delta$ & 0.4848 & $0.4749_{-0.24}^{+0.39}$ & -0.1248 & 0.9998 \\
$w$ & -1.866 & $-2.004_{-0.58}^{+0.59}$ & -2.995 & -1.142 \\
$\Omega_{m 0}$ & 0.436 & $0.4339_{-0.094}^{+0.098}$ & 0.2545 & 0.6134 \\
\hline \hline
\end{tabular}

TABLE II: Summary of the best fit values and main results for the free parameters of the scenario of interacting dark energy, using only $\mathrm{CC}+H_{0}$ observational data. The parameter $\Omega_{m}$ is the contribution of baryons plus dark matter, namely $\Omega_{m}=$ $\Omega_{b}+\Omega_{c d m}$.

\begin{tabular}{ccccc}
\hline \hline Param. & best-fit & mean $\pm \sigma$ & $95 \%$ lower & $95 \%$ upper \\
\hline \hline$\Omega_{c d m 0}$ & 0.2246 & $0.2229_{-0.0069}^{+0.0063}$ & 0.2099 & 0.2365 \\
$H_{0}$ & 71.17 & $71.37_{-1.3}^{+1.3}$ & 68.67 & 74.01 \\
$\delta$ & 0.00099 & $0.00196_{-0.0046}^{+0.0038}$ & -0.00631 & 0.01085 \\
$w$ & -1.085 & $-1.087_{-0.028}^{+0.027}$ & -1.139 & -1.032 \\
$\alpha$ & 0.143 & $0.1422_{-0.0067}^{+0.0065}$ & 0.1291 & 0.1556 \\
$\beta$ & 3.117 & $3.126_{-0.083}^{+0.079}$ & 2.966 & 3.29 \\
$M$ & -19.04 & $-19.04_{-0.037}^{+0.041}$ & -19.12 & -18.96 \\
$\Delta_{M}$ & -0.0721 & $-0.0680_{-0.023}^{+0.024}$ & -0.116 & -0.0211 \\
$\Omega_{m 0}$ & 0.2746 & $0.2729_{-0.0069}^{+0.0063}$ & 0.2599 & 0.2865 \\
\hline \hline
\end{tabular}

TABLE III: Summary of the best fit values and main results for the free parameters of the scenario of interacting dark energy, using $\mathrm{CC}+H_{0}+\mathrm{SNeIa} / \mathrm{JLA}+\mathrm{BAO}+\mathrm{CMB}$ observational data. The parameters $\alpha, \beta, M$, and $\Delta_{M}$ are nuisance parameters as explained in subsection IIIB

the universe expansion history have a slight preference towards a cosmological scenario of interacting dark energy. This is the main result of the present investigation, and it is in agreement with the results of other observational works [9], however it has been arisen through the novel use of the recently released cosmic chronometers data.

\section{FINAL REMARKS}

In the present work we have extracted observational constraints on the scenario of interacting dark energy, without the need to consider a specific interaction form, using the recently released cosmic chronometers data. In particular, considering a general direct interaction between dark matter and dark energy sectors has the general effect of altering the evolution of the former to $\rho_{c d m} \propto a^{-3+\delta}$, where $\delta$ is the parameter that quantifies the deviation from the non-interacting case. Hence, one can use observational data in order to fit $\delta$ as well as the other cosmological parameters.

In our analysis we used (i) the very recently released 

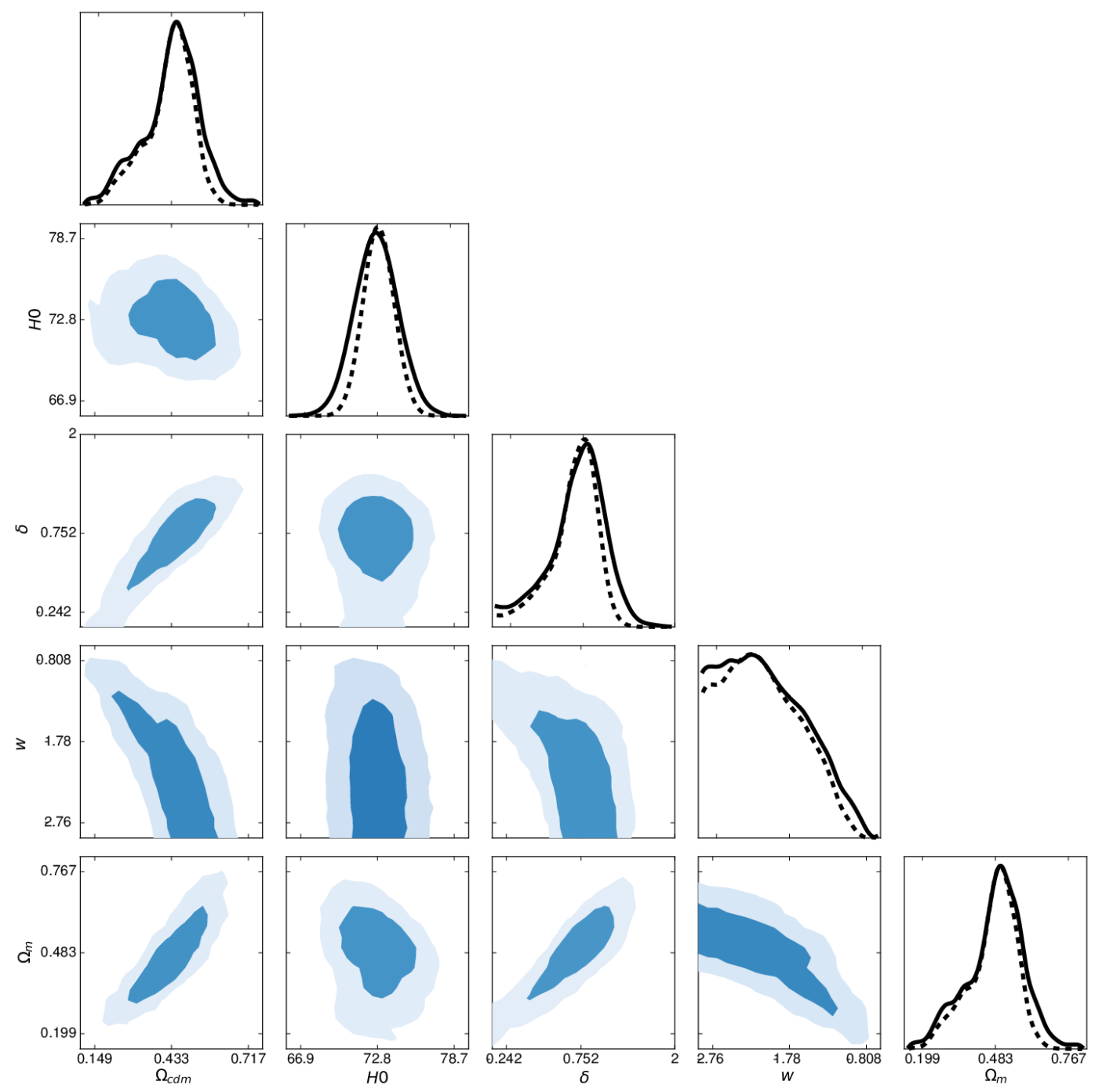

FIG. 3: $\quad 68.3 \%$ and 95.4\% confidence-level contour plots for the free parameters of the scenario of interacting dark energy, using only $\mathrm{CC}+\mathrm{H}_{0}$ observational data. Additionally, we depict the marginalized one-dimensional posterior distribution, where the dashed curve stands for the average likelihood distribution.

cosmic chronometer data sets along with the very latest measured value of the local Hubble parameter, $H_{0}=$ $73.02 \pm 1.79 \mathrm{~km} / \mathrm{s} / \mathrm{Mpc}$ [41, (ii) "joint light curves" (JLA) sample containing 740 latest Supernovae Type Ia data points, (iii) baryon acoustic oscillation data points, (iv) CMB distance priors adopting 2015 Planck TT, TE, $\mathrm{EE}+$ lowP data, marginalized over the amplitude of the lensing power spectrum 52 . We presented two different sets of values of the model parameters, one arising using the combined set of $\mathrm{CC}+\mathrm{H}_{0}$ data (summarized in Fig. 3 and Table II), and one arising using the $\mathrm{CC}+\mathrm{H}_{0}+$ $\mathrm{SNeIa} / \mathrm{JLA}+\mathrm{BAO}+\mathrm{CMB}$ data (summarized in Fig. 4 and Table III].

We found that the combined analysis $C C+H_{0}$ slightly favors a non-zero value for the interacting parameter $\delta$, while the dark energy equation-of-state parameter lies below -1 . Additionally, for the combined analysis, using
$\mathrm{CC}+\mathrm{H}_{0}+\mathrm{SNeIa} / \mathrm{JLA}+\mathrm{BAO}+\mathrm{CMB}$ data, we found that $\delta$ is close to zero, nevertheless the possibility of a small interaction is not ruled out, and moreover we find that the dark energy equation-of-state parameter lies below -1 at $3 \sigma$ confidence level.

In summary, using the latest observational data, we have found that an interaction between dark matter and dark energy sectors is mildly favored.

\section{Acknowledgments}

S.P. acknowledges Science and Engineering Research Board (SERB), Govt. of India, for awarding the National Post-Doctoral Fellowship (File No: PDF/2015/000640). This article is based upon work from COST Action "Cosmology and Astrophysics Network for Theoretical Ad- 


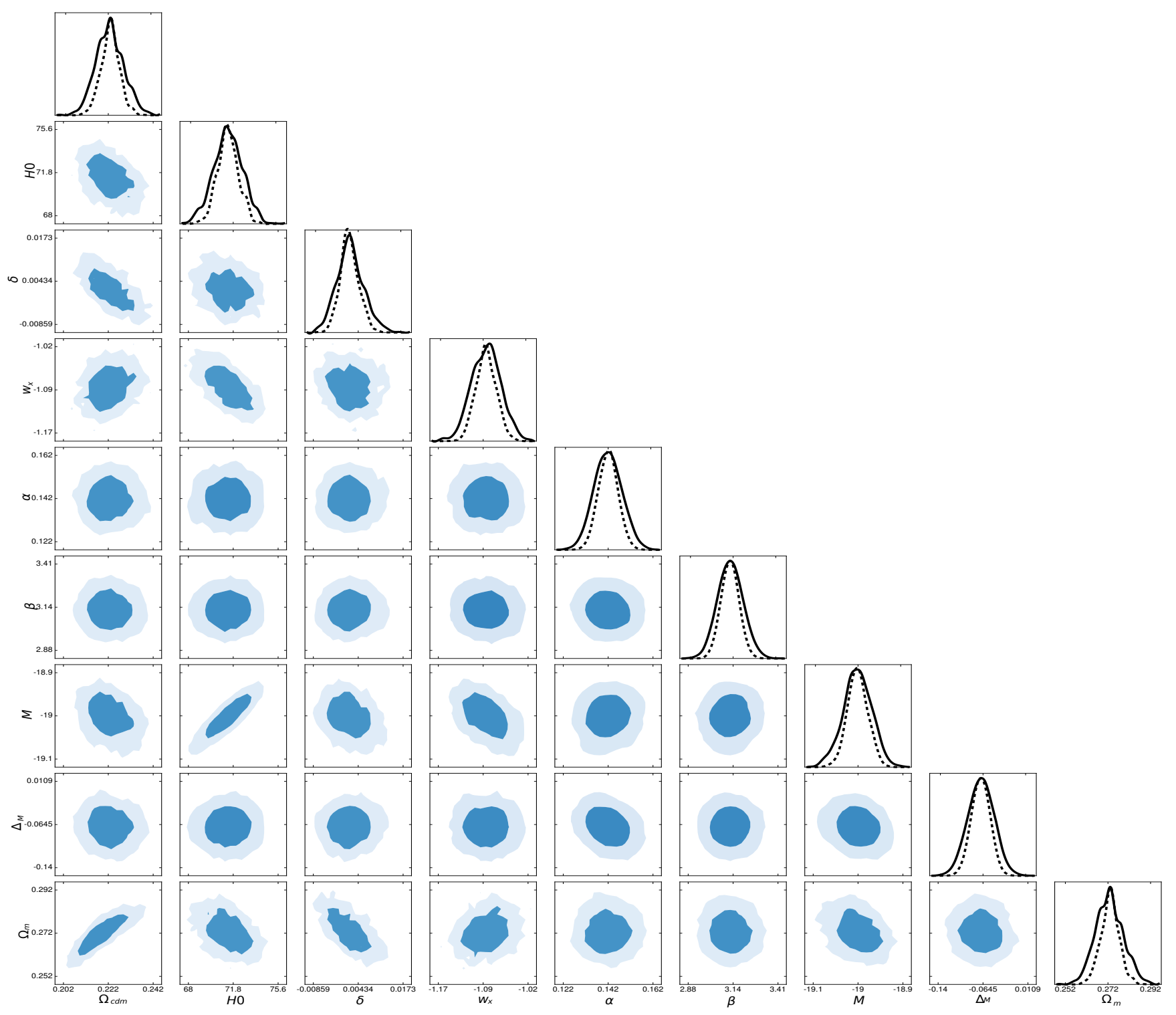

FIG. 4: $\quad 68.3 \%$ and 95.4\% confidence-level contour plots for the free parameters of the scenario of interacting dark energy, using $C C+H_{0}+S N e I a / J L A+B A O+C M B$ observational data. Additionally, we depict the marginalized one-dimensional posterior distribution, where the dashed curve stands for the average likelihood distribution.

vances and Training Actions", supported by COST (European Cooperation in Science and Technology). The authors thank N. Tamanini for useful comments. Finally, the authors gratefully acknowledge an anonymous referee for his/her valuable comments.
[1] Planck collaboration, arXiv:1502.01589 [astro-ph.CO].

[2] P. J. Steinhardt, Phil. Trans. Roy. Soc. Lond. A 361, 2497 (2003).

[3] E. J. Copeland, M. Sami and S. Tsujikawa, Int. J. Mod. Phys. D 15, 1753 (2006).

[4] Y. F. Cai, E. N. Saridakis, M. R. Setare and J. Q. Xia, Phys. Rept. 493, 1 (2010).

[5] S. Nojiri and S. D. Odintsov, Phys. Rept. 505, 59 (2011).

[6] S. Capozziello and M. De Laurentis, Phys. Rept. 509,
167 (2011).

[7] Yu. L. Bolotin, A. Kostenko, O. A. Lemets and D.A. Yerokhin, Int. J. Mod. Phys. D 24, 1530007 (2015).

[8] B. Wang, E. Abdalla, F. Atrio-Barandela and D. Pavón, arXiv:1603.08299 [astro-ph.CO].

[9] V. Salvatelli, N. Said, M. Bruni, A. Melchiorri and D. Wands, Phys. Rev. Lett. 113, no. 18, 181301 (2014).

[10] J. Solà, A. Gomez-Valent and J. d. C. Perez, arXiv:1602.02103 [astro-ph.CO]. 
[11] J. Solà, A. Gomez-Valent and J. de Cruz Prez, Astrophys. J. 811, L14 (2015).

[12] J. Solà, A. Gomez-Valent and J. de Cruz Prez, Rafael C. Nunes, arXiv:1606.00450 [gr-qc].

[13] J. H. He, B. Wang and E. Abdalla, Phys. Rev. D 83, 063515 (2011).

[14] X. D. Xu, B. Wang, P. Zhang and F. Atrio-Barandela, JCAP 1312, 001 (2013).

[15] A. A. Costa, X. D. Xu, B. Wang, E. G. M. Ferreira and E. Abdalla, Phys. Rev. D 89 (2014) no.10, 103531.

[16] E. J. Ruiz and D. Huterer, Phys. Rev. D 91, 063009 (2015).

[17] T. Yang, Z. K. Guo and R. G. Cai, Phys. Rev. D 91, no. 12,123533 (2015).

[18] Y. H. Li, J. F. Zhang and X. Zhang, Phys. Rev. D 93, no. 2, 023002 (2016).

[19] M. G. Richarte and L. Xu, arXiv:1506.02518 [astroph.CO].

[20] J. Väliviita and E. Palmgren, JCAP 1507, 015 (2015).

[21] M. Eingorn and C. Kiefer, JCAP 1507, 036 (2015).

[22] S. Pan, S. Bhattacharya and S. Chakraborty, Mon. Not. Roy. Astron. Soc. 452, 3038 (2015).

[23] R. Murgia, S. Gariazzo and N. Fornengo, JCAP 1604, no. 04, 014 (2016).

[24] P. Wang and X. H. Meng, Class. Quant. Grav. 22, 283 (2005).

[25] F. E. M. Costa, E. M. Barboza, Jr. and J. S. Alcaniz, Phys. Rev. D 79, 127302 (2009).

[26] R. C. Nunes and E. M. Barboza Jr., Gen. Relt. Grav. 46, 1820 (2014).

[27] X. m. Chen, Y. Gong, E. N. Saridakis and Y. Gong, Int. J. Theor. Phys. 53, 469 (2014).

[28] O. Bertolami, Nuovo Cim. B 93, 36 (1986).

[29] K. Freese, F. C. Adams, J. A. Frieman and E. Mottola, Nucl. Phys. B 287, 797 (1987).

[30] J. C. Carvalho, J. A. S. Lima and I. Waga, Phys. Rev. D 46, 2404 (1992).

[31] M. S. Berman, Phys. Rev. D 43, 1075 (1991).

[32] D. Pavón, Phys. Rev. D 43, 375 (1991).

[33] D. Wands, J. De-Santiago and Y. Wang, Class. Quant. Grav. 29, 145017 (2012).

[34] J. Solà, J. Phys. Conf. Ser. 453, 012015 (2013).

[35] R. Jimenez and A. Loeb, Astrophys. J. 573, 37 (2002).
[36] M. Moresco et al., arXiv:1601.01701 [astro-ph.CO].

[37] M. Moresco, MNRAS, 450, 16 (2015).

[38] J. Simon, L. Verde, and R. Jimenez, Phys. Rev. D 71, 123001 (2005).

[39] D. Stern, R. Jimenez, L. Verde, M. Kamionkowski, and S. A. Stanford, JCAP, 1002, 008 (2010).

[40] C. Zhang, H. Zhang, S. Yuan, T. J. Zhang and Y. C. Sun, Res. Astron. Astrophys. 14, no. 10, 1221 (2014).

[41] A. G. Riess et al., arXiv:1604.01424 [astro-ph.CO].

[42] A. G. Riess et al., (Supernoave Search Team Collaboration), Astron. J. 116, 1009 (1998).

[43] S. Perlmutter et al., (Supernoave Cosmology Project) Astrophys. J. 517, 565 (1999).

[44] M. Betoule et al., (SDSS collaboration), Astron. Astrophys. 568, A22 (2014).

[45] F. Beutler, C. Blake, M. Colless, D. H. Jones, L. StaveleySmith, L. Campbell, Q. Parker, W. Saunders and F. Watson, MNRAS 416, 3017 (2011).

[46] A. J. Ross, L. Samushia, C. Howlett, W. J. Percival, A. Burden and M. Manera, MNRAS 449, 835 (2015).

[47] L. Anderson et al., MNRAS 441, 24 (2014).

[48] A. Font-Ribera, et al., JCAP 1405, 027 (2014).

[49] J. R. Bond, G. Efstathiou and M. Tegmark, Mon. Not. Roy. Astron. Soc. 291, L33 (1997).

[50] G. Efstathiou and J. R. Bond, Mon. Not. Roy. Astron. Soc. 304, 75 (1999).

[51] Y. Wang and P. Mukherjee, Phys. Rev. D 76, 103533 (2007).

[52] Q. G. Huang, K. Wang and S. Wang, JCAP 1512 (2015) $12,022$.

[53] D. Blas, J. Lesgourgues, and T. Tram, JCAP 1107, 034 (2011).

[54] B. Audren, J. Lesgourgues, K. Benabed and S. Prunet, JCAP 1302, 001 (2013).

[55] Y. Wang, D. Wands, L. Xu, J. D. Santiago and A. Hojjati, Phys. Rev. D 87, 083503 (2013).

[56] D. Wands, J. D. Santiago and Y. Wang, Class. Quant. Grav. 29, 145017 (2012).

[57] C. P. Ma and E. Bertschinger, Astrophys. J. 7, 455 (1995).

[58] http://www.cosmos.esa.int/web/planck/pla 